\title{
A method for concentrating lipid peptide DNA and siRNA nanocomplexes that retains their structure and transfection efficiency
}

This article was published in the following Dove Press journal:

International Journal of Nanomedicine

I April 2015

Number of times this article has been viewed

\author{
Aristides D Tagalakis ${ }^{1, *}$ \\ Sara Castellaro ${ }^{1,2, *}$ \\ Haiyan Zhou' \\ Alison Bienemann ${ }^{3}$ \\ Mustafa M Munye' \\ David McCarthy ${ }^{4}$ \\ Edward A White ${ }^{3}$ \\ Stephen L Hart ${ }^{\prime}$
}

'Experimental and Personalised Medicine Section, University College London (UCL) Institute of Child Health, London, UK; ${ }^{2}$ Department of Pharmacy, University of Genova, Genova, Italy; ${ }^{3}$ Functional Neurosurgery Research Group, School of Clinical Sciences, AMBI Labs, University of Bristol, Southmead Hospital, Bristol, UK; ${ }^{4}$ UCL School of Pharmacy, London, UK

*These authors contributed equally to this work
Correspondence: Aristides D Tagalakis Experimental and Personalised Medicine Section, UCL Institute of Child Health, 30 Guilford Street, London, WCIN IEH, UK Tel +4420790528I6

Email a.tagalakis@ucl.ac.uk
Abstract: Nonviral gene and small interfering RNA (siRNA) delivery formulations are extensively used for biological and therapeutic research in cell culture experiments, but less so in in vivo and clinical research. Difficulties with formulating the nanoparticles for uniformity and stability at concentrations required for in vivo and clinical use are limiting their progression in these areas. Here, we report a simple but effective method of formulating monodisperse nanocomplexes from a ternary formulation of lipids, targeting peptides, and nucleic acids at a low starting concentration of $0.2 \mathrm{mg} / \mathrm{mL}$ of DNA, and we then increase their concentration up to $4.5 \mathrm{mg} / \mathrm{mL}$ by reverse dialysis against a concentrated polymer solution at room temperature. The nanocomplexes did not aggregate and they had maintained their biophysical properties, but, importantly, they also mediated DNA transfection and siRNA silencing in cultured cells. Moreover, concentrated anionic nanocomplexes administered by convection-enhanced delivery in the striatum showed efficient silencing of the $\beta$-secretase gene $B A C E 1$. This method of preparing nanocomplexes could probably be used to concentrate other nonviral formulations and may enable more widespread use of nanoparticles in vivo.

Keywords: nanoparticles, concentration, anionic liposome, siRNA, DNA, targeted gene delivery

\section{Introduction}

Nonviral formulations comprising mixtures of cationic polymers or lipids that selfassemble into nanoparticles upon mixing with nucleic acids have been widely used in genetic and gene therapy research since the late 1980s. More recently, these vectors have become more prevalent with the development of small interfering RNA (siRNA)mediated gene silencing. ${ }^{1-8}$ While a wide variety of formulations are used in cell culture experiments, the number of formulations in clinical trials for gene or siRNA therapies is more limited. ${ }^{8}$ The barriers to transfection in vivo ${ }^{8,9}$ are very different to the cell culture environment and not all formulations are compatible with usage in live animals. A further challenge is the technical problems associated with preparing monodisperse nanoparticle formulations in the volumes and concentrations required in vivo. ${ }^{10}$

Self-assembly of nanoparticles with cationic reagents and nucleic acids is mediated largely by electrostatic interactions. Empirically optimized ratios of these components are required to produce monodisperse nanoparticles with peak transfection efficiency. The formulation used in this study comprises a mixture of liposomes and targeting peptides that self-assemble on mixing with DNA ${ }^{11-18}$ or siRNA ${ }^{19-21}$ to form LPD or LPR receptor-targeted nanocomplexes (RTNs). Based on this platform, we have described both cationic and anionic RTN formulations and shown their in vivo potential by delivery 
to tumors, lung epithelium, brain, and blood vessels. ${ }^{11-16,18,21,22}$ The formulation of RTNs is more complicated than simple lipoplex or polyplexes and becomes increasingly problematic in our experience at higher nucleic acid concentrations of more than $0.5 \mathrm{mg} / \mathrm{mL}$ with large aggregates and precipitates forming, which leads to poor biodistribution, systemic toxicity, and low transfection efficiency in vivo. ${ }^{10}$

The RTN formulation process requires fast mixing of liposome, peptide, and nucleic acid components, which is easily achieved by rapid pipetting at low volumes and concentrations - for example, where the nucleic acid is less than $300 \mu \mathrm{g} / \mathrm{mL}$. For in vivo applications by systemic delivery, or in the brain where only very small volumes can be injected, higher concentrations of nucleic acids are required of $1 \mathrm{mg} / \mathrm{mL}$ or more. ${ }^{10,23}$ At these concentrations, nanocomplexes following pipette-based mixing methods, even in small volumes, rapidly precipitate due to instantaneous electrostatic interactions of the components. Sophisticated mixing technologies are available such as microfluidics, continuous flow systems, and in-line mixing, ${ }^{24-27}$ but each of these usually requires expensive equipment and timeconsuming optimization of the process and consumes large amounts of materials increasing costs.

Recently, Vauthier et $\mathrm{al}^{28}$ proposed a method of concentrating nanoparticles without aggregation using osmosis in a simple laboratory setup. We report here the application of this facile, small-scale method to concentrate LPD and LPR formulations, which should work equally well with liposomal or polymeric nanocomplex formulations, enhancing their utility for in vivo experiments. Nanocomplexes were evaluated for their biophysical properties, as well as for their in vitro and in vivo transfection efficiencies. This method could find widespread utility in the development of many different nucleic acid formulations for in vivo applications, potentially hastening the development of new genetic therapies.

\section{Materials and methods Materials}

Dextran (molecular weight [MW] 150,000) from Leuconostoc mesenteroides and dialysis tubing cellulose membrane MW cut-off (CO) 14,000 were obtained from Sigma-Aldrich Co. (St Louis, MO, USA). 1,2-dioleoylsn-glycero-3-phospho-(1'-rac-glycerol) (DOPG), 1,2-diO-octadecenyl-3-trimethylammonium propane (DOTMA), 1,2-dioleoyl-sn-glycero-3-phosphoethanolamine (DOPE), 1,2-dipalmitoyl-sn-glycero-3-phosphoethanolamine-N(methoxy[polyethylene glycol]-2000) (DPPE PEG2000), and DOTMA/DOPE (1:1 molar ratio) were purchased from
Avanti Polar Lipids, Inc. (Alabaster, AL, USA). Peptide Y ( $\mathrm{K}_{16}$ GACYGLPHKFCG) was synthesized by ChinaPeptides Co., Ltd. (Shanghai, People's Republic of China). Rabies virus glycoprotein targeting peptide (RVG-9R) (YTIWMPENPRPGTPCDIFTNSRGKRASNGGGGRRRRRRRRR) was synthesized by AMS Biotechnology Limited (Abingdon, UK). Silencer Firefly Luciferase (GL2 + GL3) and Silencer Negative Control \#1 siRNA were obtained from Applied Biosystems (Thermo Fisher Scientific, Waltham, MA, USA). Lipofectamine ${ }^{\circledR} 2000$ (L2K) was purchased from Invitrogen (Thermo Fisher Scientific). The siRNA for the $B A C E 1$ in vivo studies was bought from Eurofins MWG Operon LLC (Huntsville, AL, USA) and the sequences were: $B A C E 1$ (sense) 5' GCUUUGUGGAGAUGGUGGAdTdT 3'; and BACE1 (antisense) 5' UCCACCAUCUCCACAAAGCdTdT 3'. The plasmid pCI-Luc consists of the luciferase gene from pGL3 (Thermo Fisher Scientific) subcloned into pCI (Promega Corporation, Fitchburg, WI, USA). The plasmid pEGFP-N1 $(4.7 \mathrm{~kb})$ containing the gene GFP was obtained from Clontech Laboratories, Inc. (Mountain View, CA, USA).

\section{Liposome and nanoparticle formulation}

Lipid stocks were dissolved in chloroform at $10 \mathrm{mg} / \mathrm{mL}$. Lipids were then mixed at the required molar ratios in a round-bottomed flask and the chloroform was slowly evaporated in a rotary evaporator (BÜCHI Labortechnik AG, Flawil, Switzerland) to produce a lipid film. Lipids were then rehydrated with sterile, distilled water while constantly rotated overnight, and they were then sonicated in an ultrasonic water bath (Jencons-PLS, Bedfordshire, UK) to reduce their size. The anionic liposomes that were made were: DOPG:DOPE:DOPE PEG2000 (L ${ }^{\mathrm{AP}} 1$ ); and DOPG:DOPE:DPPE PEG2000 (L $\left.\mathrm{L}^{\mathrm{AP}} 2\right)$ at a molar ratio of 47.5:47.5:5 mol\%, respectively. ${ }^{16,21}$

Anionic nanocomplexes were prepared in water at a 4:3:1 molar charge ratio ${ }^{16}$ of liposome:peptide:siRNA or liposome:peptide:DNA, by adding the peptide to the siRNA (PRL) or DNA (PDL), incubating for 15 minutes at room temperature, and then adding the liposome with rapid mixing and incubating at room temperature for a further $20 \mathrm{~min}$ utes. Cationic RTN formulations (at a weight ratio of $1: 4: 1$, liposome:peptide:DNA) were made by first adding the peptide to the liposome DOTMA/DOPE, followed by addition of the DNA with rapid mixing and incubation for 30 minutes at room temperature to allow for complex formation. The nanocomplexes prepared were termed LYD (liposome DOTMA/ DOPE, peptide Y, and DNA), PRL (peptide Y or RVG-9R, 
siRNA, liposome $\mathrm{L}^{\mathrm{AP}}$ 2), and PDL (peptide $\mathrm{Y}$, DNA, liposome $\mathrm{L}^{\mathrm{AP}} 1$ ). Peptide/siRNA nanocomplexes were also made by mixing peptide RVG-9R with siRNA at a $4: 1$ weight ratio and incubation at room temperature for 30 minutes.

\section{Concentration experiments by reverse dialysis}

The dialysis tubing was boiled in a solution of $2 \%$ $\mathrm{NaHCO}_{3} / 1 \mathrm{mM}$ ethylenediaminetetraacetic acid (EDTA) for 10 minutes. It was then rinsed thoroughly with $\mathrm{ddH}_{2} \mathrm{O}$ and then boiled for 10 minutes in a solution of $1 \mathrm{mM}$ EDTA. It was then rinsed with $\mathrm{ddH}_{2} \mathrm{O}$, cooled at room temperature, and then stored at $4^{\circ} \mathrm{C}$ in a fresh solution of $1 \mathrm{mM}$ EDTA. Dextran solutions (100-300 g/L) were prepared by dissolving small amounts of dextran in sterile ddH20 by stirring and heating at $60^{\circ} \mathrm{C}$ for 30 minutes.

Then, $0.5-1.5 \mathrm{~mL}$ of nanocomplexes were prepared as described earlier at a nucleic acid concentration of 175-200 $\mu \mathrm{g} / \mathrm{mL}$. The volume of the counter-dialysis (dextran) medium was 12-14 $\mathrm{mL}$ (depending on the volume of the nanocomplexes in the tubing), and different concentrations of dextran were used: $100 \mathrm{~g} / \mathrm{L} ; 150 \mathrm{~g} / \mathrm{L} ; 200 \mathrm{~g} / \mathrm{L}$; $250 \mathrm{~g} / \mathrm{L}$; and $300 \mathrm{~g} / \mathrm{L}$. Dialysis was performed at room temperature in disposable conical centrifuge $15 \mathrm{~mL}$ tubes. At different time points, and at the end of the dialysis, the concentrated nanocomplexes were collected from the dialysis tubing and the concentration was measured in a NanoDrop spectrophotometer (Thermo Fisher Scientific), and the size and charge measurements were performed, as will be described. Each experiment was performed in triplicate.

\section{Particle size and charge measurements}

Nanocomplex preparations were diluted with distilled water to a final volume of $1 \mathrm{~mL}$ at a concentration of $5 \mu \mathrm{g} / \mathrm{mL}$ with respect to DNA or siRNA. They were then analyzed for size by intensity and charge ( $\zeta$ potential) by dynamic light scattering (DLS) using a Malvern Nano ZS Zetasizer (Malvern Instruments, Malvern, UK). The data were then processed by software provided by the manufacturer, DTS version 5.03. Size measurements with a polydispersity index (PDI) of less than 0.3 were accepted as monodisperse.

\section{In vitro transfections}

The murine neuroblastoma cell line Neuro-2A (American Type Culture Collection, Manassas, VA, USA) and Neuro2A-Luc cells, were maintained in Dulbecco's Modified Eagle's Medium, 1\% nonessential amino acids, $1 \mathrm{mM}$ sodium pyruvate, and 10\% fetal calf serum (FCS) (Thermo
Fisher Scientific). The human bronchial epithelial cells 16HBE140- (shortened to HBE) were provided by D Gruenert (San Francisco, CA, USA) and were cultured in Eagle's Minimal Essential Medium with HEPES modification (Sigma-Aldrich Co.), 10\% FCS, and $2 \mathrm{mM}$ L-glutamine. All cells were maintained at $37^{\circ} \mathrm{C}$ in a humidified atmosphere in $5 \%$ carbon dioxide. Cells were seeded in 96-well plates at $2 \times 10^{4}$ cells per well 24 hours prior to transfection. Following the removal of growth medium, $200 \mu \mathrm{L}$ of complexes that underwent concentration were diluted in OptiMEM in order to contain $0.25 \mu \mathrm{g}$ of plasmid DNA or $50 \mathrm{nM}$ siRNA, and they were added to the cells in replicates of six. The same amount of DNA or siRNA was added per well when the nanocomplexes were made fresh (ie, not concentrated). Plates were centrifuged at $1,500 \mathrm{rpm}$ for 5 minutes $(400 \times \mathrm{g})$ and incubated for 4 hours at $37^{\circ} \mathrm{C}$, then transfection medium was replaced by the complete growth medium and incubated for a further 24 hours. Luciferase expression was measured in cell lysates with a luciferase assay (Promega Corporation) in a FLUOstar OPTIMA luminometer (BMG LABTECH GmbH, Ortenberg, Germany). The amount of protein present in each sample was determined with the Bio-Rad protein assay reagent (BioRad Laboratories Inc., Hercules, CA, USA) in a FLUOstar OPTIMA luminometer. Luciferase activity was expressed as relative light units per milligram of protein (RLU/mg). Each measurement was performed in groups of six and the mean was determined.

Neuro-2A cells were seeded in 96-well plates at $1.2 \times 10^{4}$ cells per well 24 hours prior to transfection with $175 \mu \mathrm{L}$ of complete serum-containing media. Twenty-four hours later, $25 \mu \mathrm{L}$ of concentrated PDL complexes were diluted in OptiMEM to contain $0.25 \mu \mathrm{g}$ of green fluorescent protein (GFP) plasmid DNA and then added to the cells in replicates of six. Plates were centrifuged at 1,500 rpm for 5 minutes $(400 \times g)$ and incubated for 48 hours at $37^{\circ} \mathrm{C}$. They were then imaged (20× magnification) using an Olympus IX70 fluorescent microscope (Olympus Corporation, Tokyo, Japan).

\section{Transmission electron microscopy (TEM)}

Copper grids (300-mesh) coated with a Formvar/carbon support film (Agar Scientific Ltd, Stansted, UK) were prepared by glow discharge in an Emitech K350G system (Emitech LTD, Nicosia, Cyprus). Nanocomplex preparations were applied to grids as a droplet then after 5 seconds, the grid was dried by blotting with filter paper. The sample was then negatively stained with $1 \%$ uranyl acetate for $2-3 \mathrm{sec}-$ onds, before blotting with filter paper and air-dried. Imaging 
was performed with a Philips CM120 BioTwin TEM and operated at an accelerating voltage of $120 \mathrm{kV}$. The images were captured using an AMT 5 MP digital TEM camera (Deben UK Limited, Suffolk, UK).

\section{Cell proliferation assay}

Cell viability was assessed using the CellTiter $96^{\circledR}$ Aqueous One Solution Cell Proliferation Assay (Promega Corporation). Neuro-2A cells were seeded in 96-well plates and transfected with anionic and cationic concentrated nanocomplexes that were diluted in OptiMEM, as discussed earlier. After 24 hours, the media were changed with growth media containing $20 \mu \mathrm{L}$ of CellTiter 96 Aqueous One Solution reagent. Finally, after incubation for 2 hours, absorbance was measured at $490 \mathrm{~nm}$ on a FLUOstar OPTIMA spectrophotometer (BMG LABTECH $\mathrm{GmbH})$. Cell viability for each complex was expressed as a percentage of the viability of control cells.

\section{Ex vivo silencing study using RVG-9R peptide}

Brains were obtained from wild-type CD1 mice and cut into small pieces $(\sim 2 \mathrm{~mm} \times 2 \mathrm{~mm} \times 2 \mathrm{~mm})$ in a Petri dish under sterile conditions, then moved to a 24 -well plate by forceps. Then, $1 \mathrm{~mL}$ of DMEM ( $1 \%$ antibiotics, no serum) was added to each well and incubated at $37^{\circ} \mathrm{C}$ and transfected in triplicate, as described previously. Forty-eight hours later, the medium was removed from the culture dish and the tissues were processed for RNA extraction using the RNeasy kit according to the manufacturer's instructions (Qiagen NV, Venlo, the Netherlands).

\section{In vivo procedures}

All murine in vivo studies were performed in accordance with University College London's animal care policies and with the authority of the appropriate UK Home Office licenses. CD1 female mice (6-8 weeks old) were injected intravenously with $100 \mu \mathrm{L}$ of anionic PRL nanoparticles (with peptide RVG-9R) containing $16 \mu \mathrm{g}$ or $50 \mu \mathrm{g}$ of siRNA $(0.64-2 \mathrm{mg} / \mathrm{kg})$ in $5 \%$ dextrose. Mice were euthanized 48 hours later and their brains were placed in RN Alater (Thermo Fisher Scientific). All rat in vivo studies were performed in accordance with the University of Bristol's animal care policies and with the authority of the appropriate UK Home Office licenses. Adult male Wistar rats (Charles River Laboratories International, Inc., Wilmington, MA, USA) (225-275 g) were used for convection-enhanced delivery (CED), as described previously. ${ }^{15,21}$ A total volume of $5 \mu \mathrm{L}$ $(6 \mu \mathrm{g}$ of siRNA or $0.024 \mathrm{mg} / \mathrm{kg}$ ) of anionic PRL nanoparticles (with peptide RVG-9R) in 5\% dextrose was delivered to the striatum at an infusion rate of $2.5 \mu \mathrm{L} /$ minute. Rats were euthanized 48 hours later, and the striata were placed in RNAlater (Thermo Fisher Scientific).

\section{qRT-PCR}

Total RNA was extracted from rat brain using the RNeasy kit according to the manufacturer's instructions (Qiagen NV). RNA was checked for integrity using the Agilent 2100 Bioanalyzer (Agilent Technologies, Santa Clara, CA, USA). All RNA samples had an RNA integrity number of more than 8 , indicating high-quality RNA. Prior to reverse transcription, each RNA sample was treated with DNase (Thermo Fisher Scientific). First-strand complementary DNA was synthesized from $1 \mu \mathrm{g}$ of DNase-treated RNA, using random hexamers, and Superscript II reverse transcriptase (Thermo Fisher Scientific) in a 1 -hour reaction at $37^{\circ} \mathrm{C}$. Rat BACE1 and rat beta-actin complementary DNAs were then quantified using Taqman primers and probes (Rn00569988_m1 and Rn00667869_m1, respectively; Thermo Fisher Scientific) and an ABI PRISM ${ }^{\circledR} 7000$ Sequence Detection System (Applied Biosystems; Thermo Fisher Scientific). The quantitative reverse transcription polymerase chain reaction (qRTPCR) assay conditions were: stage $1,50^{\circ} \mathrm{C}$ for 2 minutes; stage $2,95^{\circ} \mathrm{C}$ for 10 minutes; stage $3,95^{\circ} \mathrm{C}$ for 15 seconds, then $60^{\circ} \mathrm{C}$ for 1 minute; repeated 40 times.

\section{Western blot}

Protein gel electrophoresis and Western blot analysis were performed as described previously. ${ }^{21}$ Briefly, total protein was extracted from mouse brain using the Precellys ${ }^{\circledR}$ Homogenizer (Stretton Scientific Ltd, Derbyshire, UK). Forty micrograms of protein were loaded and separated using NuPAGE ${ }^{\circledR}$ Precast gels (10\% Bis-Tris; Thermo Fisher Scientific) and then transferred electrophoretically to a polyvinylidene fluoride membrane (Thermo Fisher Scientific). The primary antibodies used in this study were rabbit anti-BACE1 polyclonal antibody (EE-17, 1:1,000; SigmaAldrich Co.) and mouse anti- $\beta$-tubulin monoclonal antibody (1:5,000; Sigma-Aldrich Co.), and the secondary antibodies were a horseradish peroxidase-conjugated antimouse or antirabbit immunoglobulin G (Stratech Scientific Ltd, Suffolk, UK) (1:50,000). Semiquantification of the bands was performed by densitometry using the ImageJ software.

\section{Statistical analysis}

The data presented in this study are expressed as the mean \pm standard deviation and were analyzed using a two-tailed, 

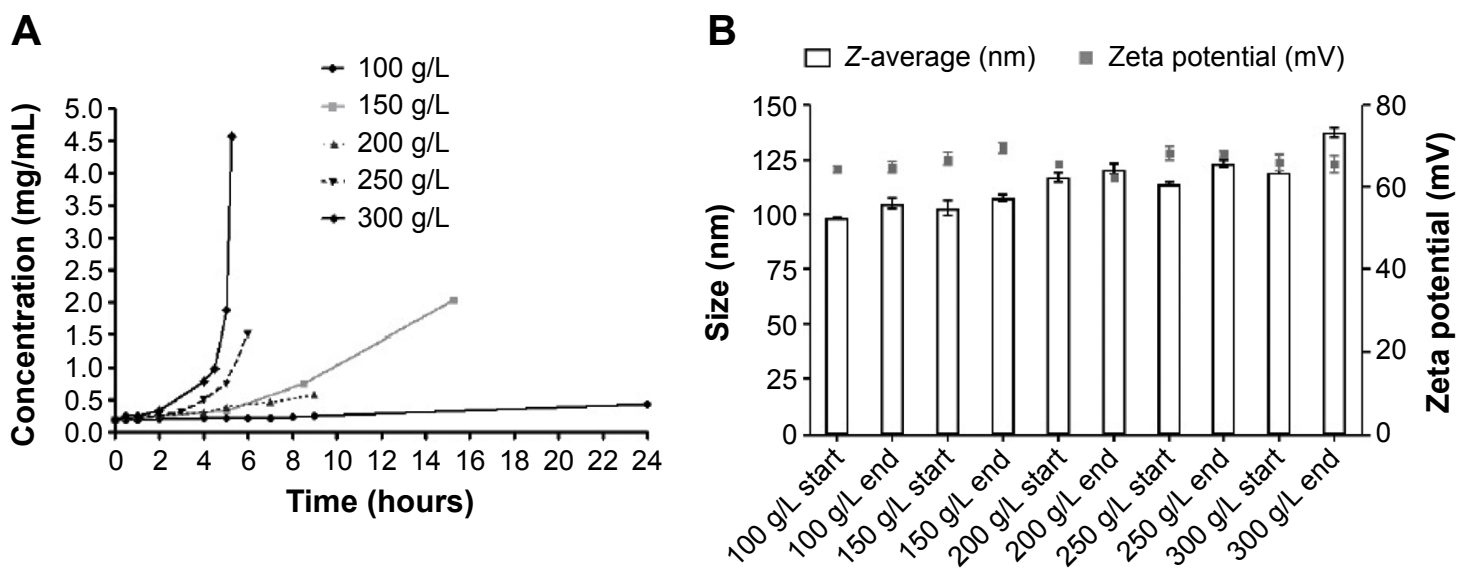

Figure I Effect of different concentrations of dextran on concentrating cationic LYD nanoparticles and on size and zeta potential.

Notes: (A) Kinetics of the concentration of LYD nanoparticles over time when varying the concentration of dextran ( $100-300 \mathrm{~g} / \mathrm{L})$. (B) Size and charge measurements of LYD nanoparticles by dynamic light scattering before and after concentration with different amounts of dextran ( $100-300 \mathrm{~g} / \mathrm{L})$.

Abbreviation: LYD, liposome I,2-di-O-octadecenyl-3-trimethylammonium propane (DOTMA)/l,2-dioleoyl-sn-glycero-3-phosphoethanolamine (DOPE), peptide Y, and DNA

unpaired Student's $t$-test or one-way analysis of variance and Bonferroni's post hoc analysis, where applicable.

\section{Results and discussion Concentrating nanoparticles and biophysical characterization}

Concentration of LYD nanoparticles ${ }^{19}$ by reverse dialysis was first performed through cellulose dialysis membrane (MWCO: 14,000) against solutions of dextran (MW: 150,000) dissolved in a range of concentrations. The high osmotic pressure of the dextran solutions leads to the displacement of water from the tubing into the surrounding dextran solution. Figure 1A shows the change in concentration of nanocomplexes achieved over time from a starting concentration of $0.2 \mathrm{mg} / \mathrm{mL}$ with respect to the DNA with varying dextran concentrations. The lowest dextran concentration of $100 \mathrm{~g} / \mathrm{L}$ resulted in a $30 \%$ increase in nanocomplex concentration after 9 hours of dialysis, whereas the $150 \mathrm{~g} / \mathrm{L}, 200 \mathrm{~g} / \mathrm{L}, 250 \mathrm{~g} / \mathrm{L}$, and $300 \mathrm{~g} / \mathrm{L}$ concentrations resulted in 3.9-fold (in 8.5 hours), 2.7-fold (in 9 hours), 8.2-fold (in 6 hours), and 23.9-fold (in 5 hours and 15 minutes) increases, respectively. When longer time points were assessed for dextran at $100 \mathrm{~g} / \mathrm{L}$ ( 24 hours) and $150 \mathrm{~g} / \mathrm{L}$ (15 hours and 15 minutes), 2.25-fold and 10.6-fold increases in DNA concentration were achieved, respectively. At dextran concentrations of $>150 \mathrm{~g} / \mathrm{L}$ nanoparticle concentrations of $0.59-4.6 \mathrm{mg} / \mathrm{mL}$ were achieved, which is in the target range for possible clinical applications. ${ }^{10,29}$

The effect of the starting volume at three different concentrations of dextran $(100 \mathrm{~g} / \mathrm{L}, 200 \mathrm{~g} / \mathrm{L}$, and $300 \mathrm{~g} / \mathrm{L})$ was investigated for its effects on the rate of concentration increase. Table 1 shows that smaller starting volumes were concentrated more quickly. For example, at $300 \mathrm{~g} / \mathrm{L}$, a $\sim 9$-fold concentration of DNA in a $0.5 \mathrm{~mL}$ formulation was achieved in only 2.2 hours, while 4.5 hours were required for the equivalent $1.5 \mathrm{~mL}$ formulation (Figure 1A). Therefore, the osmotic pressure applied to the nanoparticle solution could be controlled through the dextran concentration in the counter-dialyzing solution, which significantly affected the rate of water transfer between the two compartments.

The sizes and zeta potentials (Figure 1B) of LYD nanocomplexes were determined before and after concentrating with different dextran solutions (100-300 g/L). There was no statistical difference in either the size or the zeta potential before and after concentration (size ranges: 98-137 $\mathrm{nm}$ and zeta potential: $+64-69 \mathrm{mV}$ ), indicating that this procedure does not alter these biophysical properties. All PDI measurements before and after concentration

Table I Starting nanoparticle volumes, dextran concentrations used, total time of concentration, and concentration factors achieved for LYD formulations

\begin{tabular}{llll}
\hline $\begin{array}{l}\text { Volume } \\
(\mathbf{m L})\end{array}$ & $\begin{array}{l}\text { Dextran concentration } \\
(\mathbf{g} / \mathbf{L})\end{array}$ & $\begin{array}{l}\text { Time } \\
\text { (hours) }\end{array}$ & $\begin{array}{l}\text { Fold } \\
\text { concentration }\end{array}$ \\
\hline 1.5 & 100 & 24 & 2.2 \\
1.0 & 100 & 24 & 2.1 \\
0.5 & 100 & 9 & 2.6 \\
1.5 & 200 & 9 & 2.7 \\
1.0 & 200 & 3.75 & 2.6 \\
0.5 & 200 & 3.25 & 3.2 \\
1.5 & 300 & 5.15 & 23.9 \\
1.0 & 300 & 4 & 20.2 \\
0.5 & 300 & 2.2 & 8.9 \\
\hline
\end{tabular}

Abbreviation: LYD, liposome I,2-di-O-octadecenyl-3-trimethylammonium propane (DOTMA)/I,2-dioleoyl-sn-glycero-3-phosphoethanolamine (DOPE), peptide Y, and DNA. 
were $<0.3$, indicating monodisperse nanoparticle populations. Cationic (LYD) and anionic (PDL and PRL) nanoparticle formulations ${ }^{16,21}$ were further characterized by negative staining TEM to determine their shape and morphology before and after concentration using $300 \mathrm{~g} / \mathrm{L}$ of dextran (Figure 2). Most nanocomplexes were spheres (Figure 2A-C), but with some rods (in LYD and PDL; Figure 2A and B) and some toroidal structures (Figure $2 \mathrm{~A}$ and $\mathrm{B}$ ). The majority of the spherical particles measured by TEM for each formulation were in the range determined by DLS with no obvious differences between formulations before and after concentration.
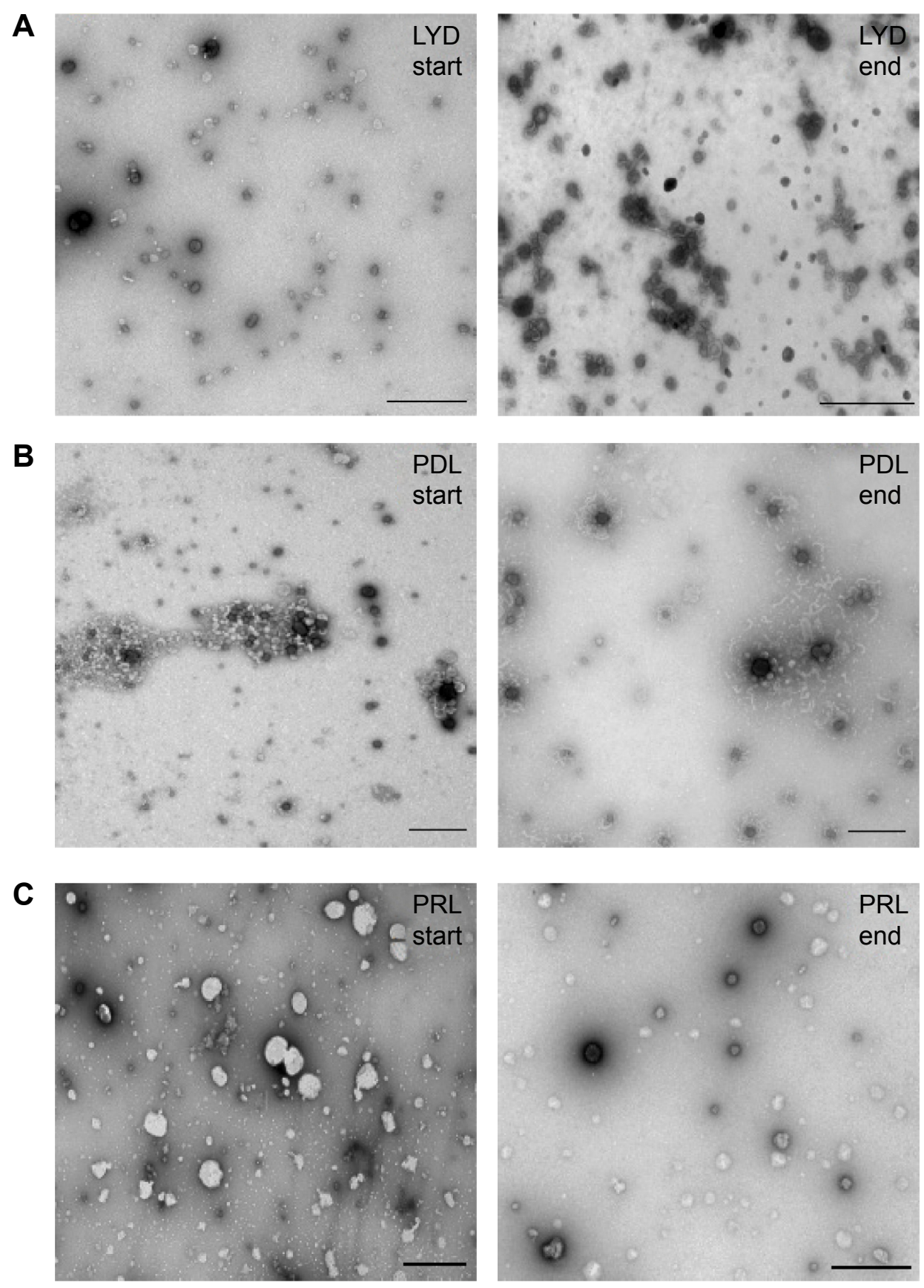

Figure 2 Electron microscopy of nanocomplexes.

Notes: Negative staining transmission electron microscopy was used to visualize (A) LYD nanoparticles before and after concentration, (B) PDL nanoparticles before and after concentration, and (C) PRL nanoparticles before and after concentration. Scale bar $=500 \mathrm{~nm}$ for all nanoparticles. $300 \mathrm{~g} / \mathrm{L}$ dextran was used to concentrate all three different nanoparticle formulations.

Abbreviations: LYD, liposome 1,2-di-O-octadecenyl-3-trimethylammonium propane (DOTMA)/I,2-dioleoyl-sn-glycero-3-phosphoethanolamine (DOPE), peptide Y, and DNA; PDL, peptide Y, DNA, liposome LAPI; PRL, peptide Y or RVG-9R, siRNA, liposome LAP2. 
This method of concentration has several advantages, as it preserves the biophysical characteristics of the nanocomplexes, avoids aggregation of the nanoparticles, and is a quick and simple method that does not need any specialized equipment. ${ }^{28}$ Based on the efficacy of the concentration, the short but predictable time required, and the fact that there were no major biophysical changes to the nanocomplexes before and after, we decided to use a dextran concentration of $300 \mathrm{~g} / \mathrm{L}$ in ongoing experiments (unless otherwise stated).
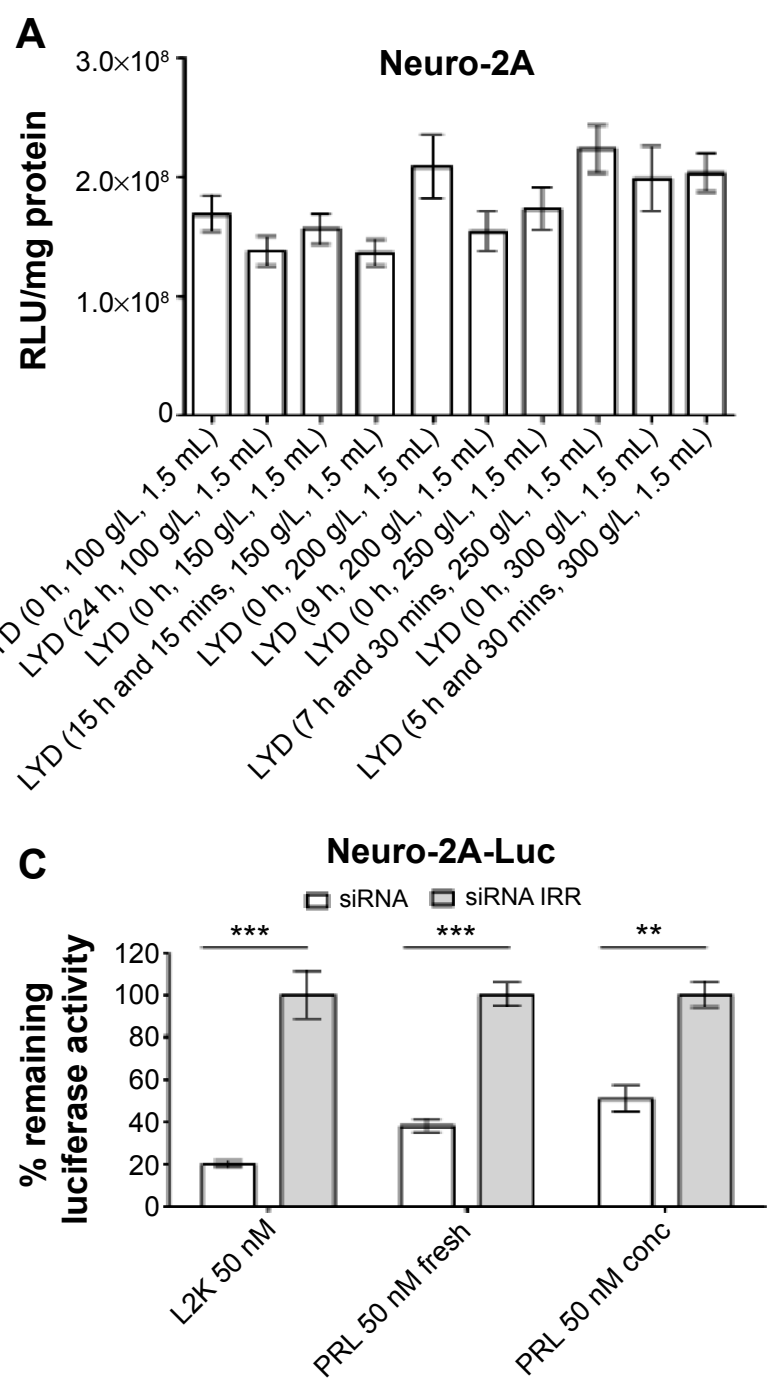

\section{Concentration of LPD nanoparticles maintains their functional abilities}

LYD nanoparticles were concentrated using different amounts of dextran (100-300 g/L), and were then used for the transfection of Neuro-2A cells (Figure 3A). There was no statistical difference in the transfection efficiency between the formulations irrespective of the amount of dextran used, or if they were concentrated or nonconcentrated. The same was true when the LYD nanoparticles that were concentrated against $300 \mathrm{~g} / \mathrm{L}$ of dextran were used for the transfection of HBE cells (Figure 3B).

\section{B}
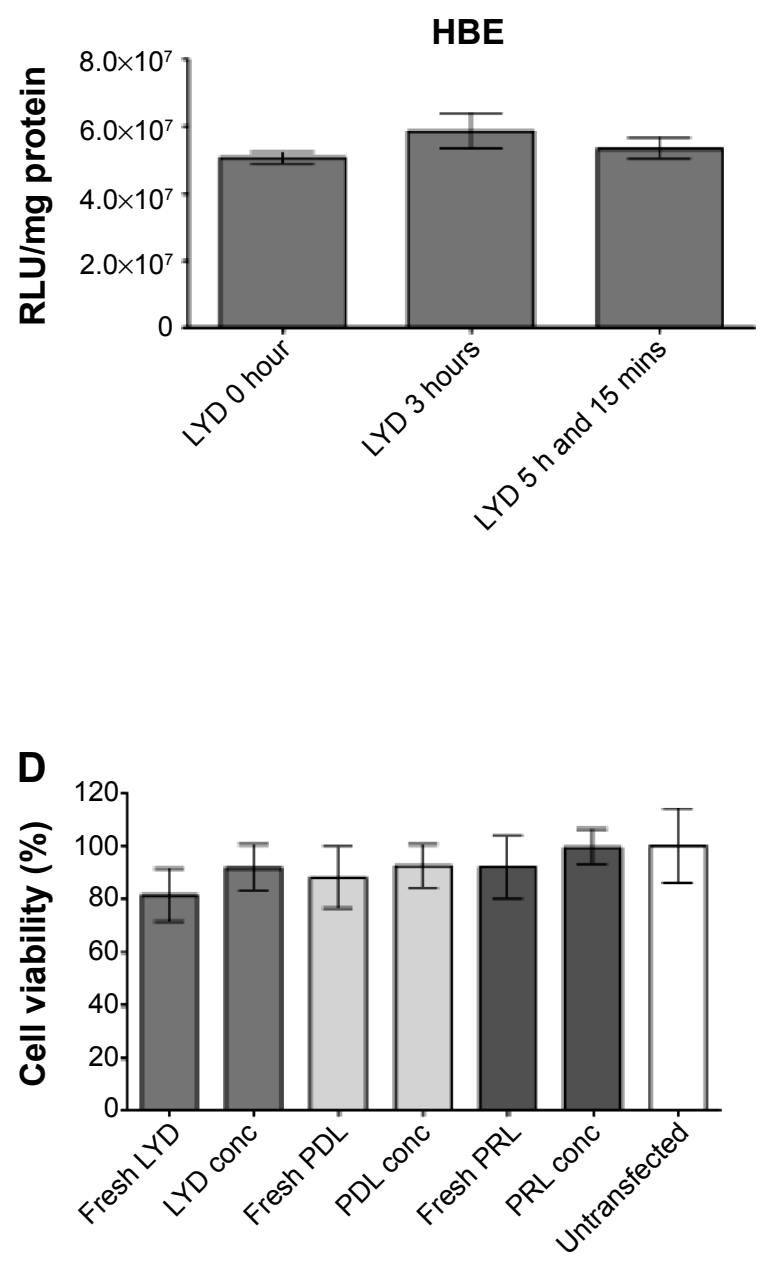

Figure 3 In vitro transfections with concentrated nanocomplexes retain transfection efficiency with lack of cytotoxicity.

Notes: (A) A total of $1.5 \mathrm{~mL}$ of LYD nanocomplexes were concentrated using different amounts of dextran (I00-300 g/L) and were used in luciferase transfections in Neuro-2A cells. (B) LYD nanoparticles before and after concentration (concentrated for 3 hours and 5 hours, 15 minutes) were used in luciferase transfections in HBE cells. (C) siRNA silencing from anionic PRL nanocomplexes (with peptide $Y$ ) before or after concentration made at a 4:3:I molar charge ratio using siRNA targeting luciferase in Neuro-2A-Luc cells at $50 \mathrm{nM}$. 24 hours later, luciferase assays were performed. L2K/siRNA nanocomplexes were used as a positive control in all in vitro silencing experiments. (D) Viability of Neuro-2A cells following transfection for 24 hours with cationic LYD and anionic PDL and PRL nanocomplexes. Cationic nanocomplexes were made at a weight ratio of I:4:I (liposome:peptide:DNA) and the anionic nanocomplexes at a molar charge ratio of 4:3:I (liposome:peptide:siRNA). The viability values were normalized to the untransfected control cells. The dextran concentration in the counter-dialyzing solution was kept constant (300 g/L) in Figure 3B-D. All transfections were performed in groups of six and mean values were calculated. Asterisks indicate comparisons of specific formulations with statistical significance $(* * P<0.0 \mathrm{I} ; * * * P<0.00 \mathrm{I})$.

Abbreviations: RLU, relative light units; LYD, liposome I,2-di-O-octadecenyl-3-trimethylammonium propane (DOTMA)/I,2-dioleoyl-sn-glycero-3-phosphoethanolamine (DOPE), peptide $Y$, and DNA; HBE, human bronchial epithelial cells I6HBEI4o-; siRNA, small interfering RNA; siRNA IRR, irrelevant control small interfering RNA; L2K, Lipofectamine ${ }^{\circledR}$ 2000; PRL, peptide Y or RVG-9R, siRNA, liposome LAP2; conc, concentrated; PDL, peptide Y, DNA, liposome LAPI; P, peptides; R, siRNA; D, DNA; h, hours. 
The concentrated, anionic PRL nanoparticles (with peptide $\mathrm{Y})^{21}$ were used for luciferase silencing of Neuro2A-Luc cells (Figure 3C). All anionic formulations resulted in significant silencing between $45 \%-70 \%$, and there was no difference between concentrated and nonconcentrated samples. The cell viability showed no particular difference between all the formulations tested, which included cationic LYD and anionic PDL and PRL made fresh or following concentration (Figure 3D).

The transfection efficiency of an anionic polyethylene glycol (PEG)ylated PDL formulation was evaluated with the plasmid expressing enhanced GFP in Neuro-2A cells. Fluorescent microscopy images provided evidence of the high transfection efficiency of PDL anionic nanoparticles following concentration (Figure 4).

\section{Ex vivo and in vivo administration of RVG-containing nanocomplexes}

We then evaluated an LPR formulation for brain delivery using RVG-9R, which binds the nicotinic acetylcholine receptor, ${ }^{30}$ formulated with anionic liposomes and siRNA against the neuronal enzyme beta-secretase (BACE1). This enzyme cleaves the amyloid precursor protein to generate amyloid-beta peptides, which are landmarks in Alzheimer's disease pathophysiology. ${ }^{31,32}$ BACE1 is therefore a main therapeutic target for Alzheimer's disease.

Ex vivo silencing studies were first performed with brain explant tissue transfected with $100 \mathrm{nM}$ siRNA in anionic PRL nanoparticles containing RVG-9R or PR nanoparticles containing RVG-9R and siRNA (RVG-9R/siRNA). The analysis of silencing by qRT-PCR revealed about $40 \%$ silencing of $B A C E 1$ with PRL nanoparticles $(P<0.05)$ and no silencing was achieved with RVG-9R/BACE1R complexes (Figure 5A).
L2K, which was used as a positive control, achieved 54\% silencing compared to its irrelevant siRNA control $(P<0.01)$. Western blotting revealed $32 \%$ silencing of BACE1 protein (Figure 5B). We then progressed to in vivo studies with just RVG-9R-targeted anionic PRL nanoparticles.

PRL nanocomplexes were then prepared for in vivo use by formulating initially at a concentration of $0.18 \mathrm{mg} / \mathrm{mL}$ BACE1 or irrelevant siRNA and then concentrated by dialysis against $300 \mathrm{~g} / \mathrm{L}$ dextran over 4.5 hours, achieving an over sixfold concentration (Figure 5C). These nanoparticles were used for all the subsequent in vivo studies in mice and rats. The size and charge of concentrated BACE1 siRNA formulations were $79.9 \pm 2.95 \mathrm{~nm}$ and $-44.8 \pm 1.5 \mathrm{mV}$, respectively, while the values for irrelevant control siRNA PRLs were similar at $73.3 \pm 4.0 \mathrm{~nm}$ and $-36.9 \pm 2.3 \mathrm{mV}$.

Concentrated nanoparticles were administered intravenously into mice, and 48 hours later, the brains were assessed for silencing. No silencing of mouse $B A C E 1$ was detected following intravenous injections (Figure 5D), unlike other reports that showed silencing even when the RVG/siRNA were delivered by intravenous injections. ${ }^{30,33-35}$ Other recent research showed nanoparticle deposition in the brain, but it did not examine the silencing effects there, ${ }^{36}$ or it assessed increased enzymatic activity following $\beta$-galactosidase administration. ${ }^{37}$ The lack of silencing following the intravenous administration of RVG-containing nanoparticles could be due to differences in both the amount of dose and the number of doses delivered, as we used a single $16 \mu \mathrm{g}$ or $50 \mu \mathrm{g}$ dose, whereas others use 50-150 $\mu \mathrm{g}$ for up to four doses. ${ }^{30,33-35}$ In addition, the nanoparticles used here are formed by self-assembly, whereas others use conjugation of the peptide by covalent bonds on the surface of the delivery vectors. ${ }^{36,37}$ However, this does not imply that our RVG
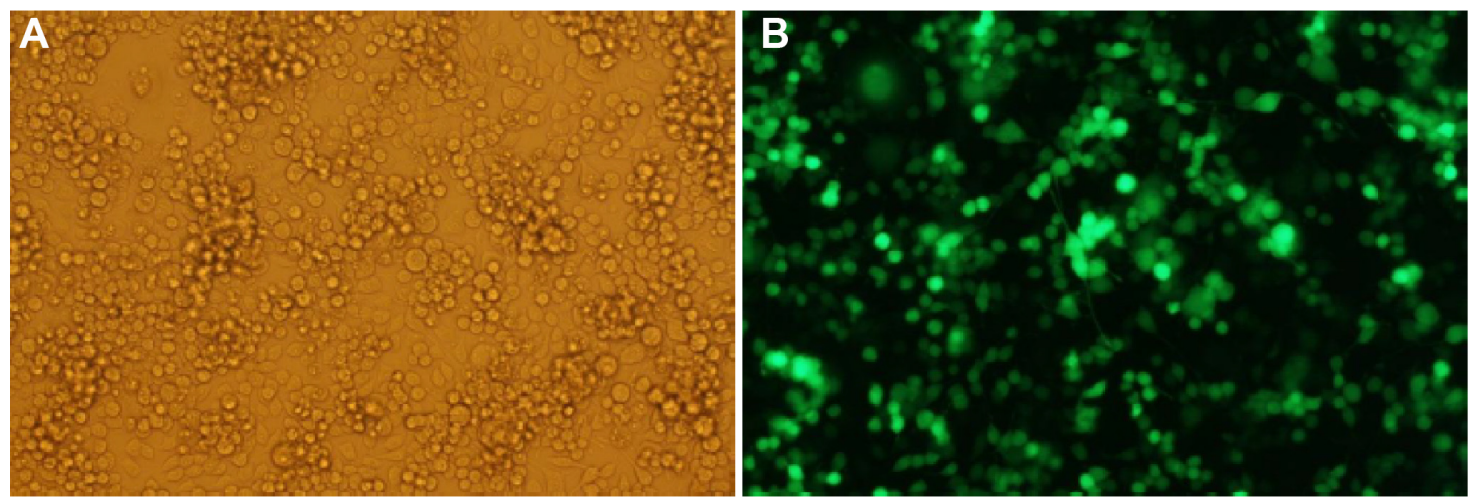

Figure 4 GFP transfection efficiency of nanocomplexes following concentration.

Notes: One anionic PRL formulation was concentrated (300 g/L dextran) and then transfected Neuro-2A cells in serum-containing media. GFP expression was observed by epifluorescence microscopy 48 hours later. Representative cells are shown in (A) phase contrast and (B) transfected cells appear green (I0X magnification). Abbreviations: GFP, green fluorescent protein; PRL, peptide $Y$ or RVG-9R, siRNA, liposome LAP2. 
A

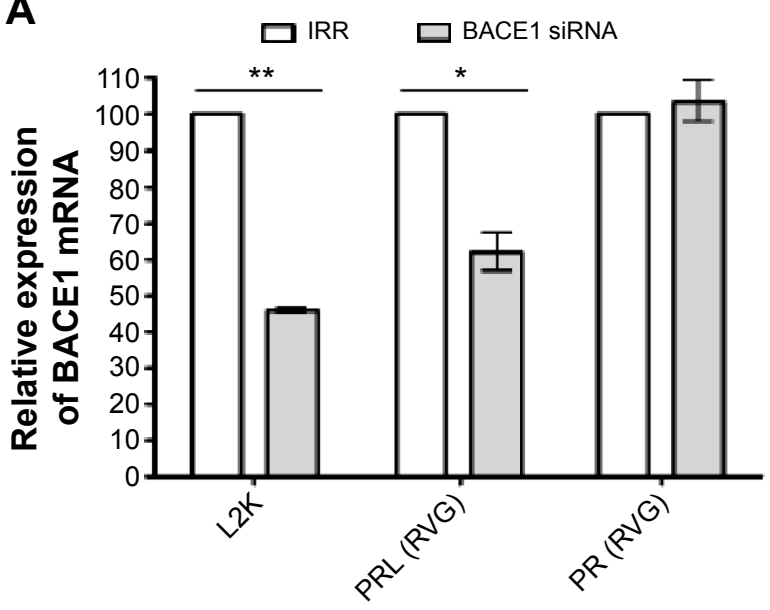

C

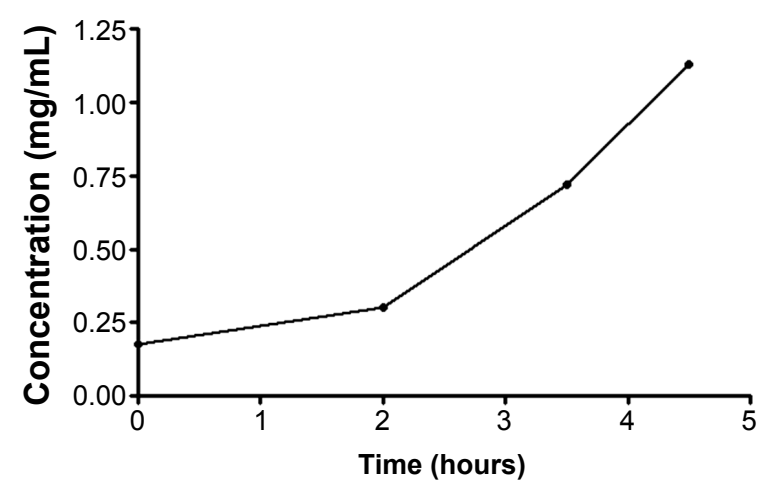

B

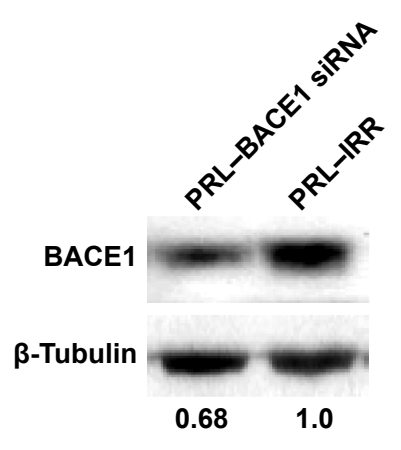

D

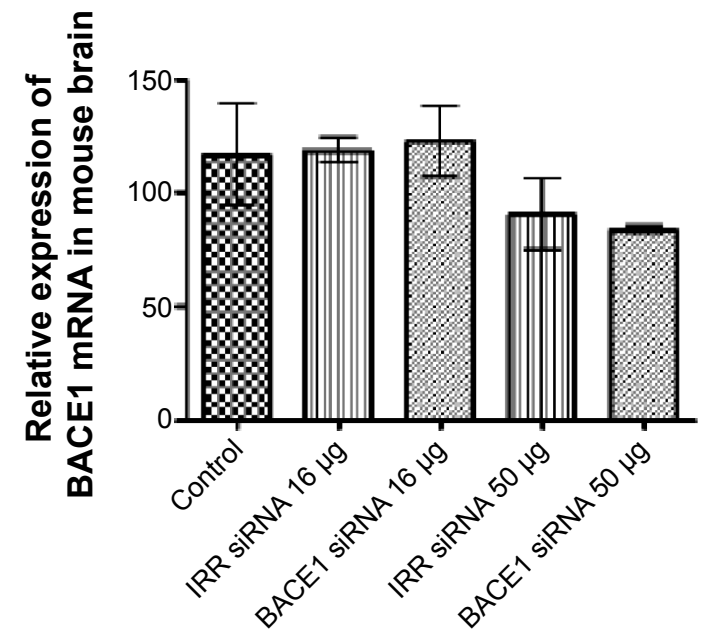

Figure 5 Nanocomplexes achieve silencing ex vivo, but not in the brain following intravenous administration.

Notes: Mice brain explants were transfected ex vivo with peptide/siRNA complexes (PR: RVG-9R/BACEI siRNA; 4:I weight ratio), anionic PEGylated PRL nanocomplexes with BACEI siRNA, L2K, or complexes with irrelevant control siRNA (all at $100 \mathrm{nM}$ ), and then 48 hours post-transfection, the tissues were processed for analysis (A) by qRT-PCR and (B) by Western blot analysis of the BACEI protein. Protein silencing was calculated with densitometric analysis using tubulin as loading control. (C) Anionic PRL nanocomplexes containing RVG-9R and BACEI siRNA were concentrated using $300 \mathrm{~g} / \mathrm{L}$ dextran over 3.5 hours, and this concentrated nanoparticle formulation was used (D) in intravenous injections. Mice were injected with $100 \mu \mathrm{L}$ of anionic PRL nanoparticles containing $16 \mu \mathrm{g}$ or $50 \mu \mathrm{g}$ BACEI siRNA or IRR siRNA, and 48 hours later, brains were processed for QRT-PCR analysis. The values are the means of three animals \pm standard deviation. Asterisks indicate comparisons of specific formulations with statistical significance $(* P<0.05 ; * * P<0.01)$.

Abbreviations: mRNA, messenger RNA; IRR, irrelevant control; siRNA, small interfering RNA; L2K, Lipofectamine ${ }^{\circledR} 2000$; PRL, peptide $Y$ or RVG-9R, siRNA, liposome LAP2; RVG, rabies virus glycoprotein targeting peptide; PR, RVG-9R/BACEI siRNA; PEG, polyethylene glycol; qRT-PCR, quantitative reverse transcription polymerase chain reaction.

peptide-containing nanoparticles did not pass the blood-brain barrier, as we did not examine their biodistribution.

We then evaluated direct brain administration in rats of anionic PRL nanoparticles (with RVG-9R) incorporating either BACE1 siRNA or irrelevant control, administered by $\mathrm{CED}^{38,39}$ into rat striata, and $B A C E 1$ expression was examined 48 hours later. A significant reduction in BACE1 mRNA was observed (Figure 6) between the $B A C E 1$-treated group and the control groups $(P<0.05$ compared to the irrelevant control group; $P<0.01$ compared to the saline group; and $P<0.001$ compared to the untreated control group).

Another study also performed direct brain administrations rather than using the intravenous route with RVG/siRNA complexes, and these showed good brain biodistribution and silencing of their target gene. ${ }^{40}$ The direct method avoids the problems of circulation clearance by the first-pass organs and the reticuloendothelial system, and requires less siRNA compared to the reported $50 \mu \mathrm{g}^{30,34,35}$ or $150 \mu \mathrm{g}$ doses. ${ }^{33}$ We have previously shown that anionic nanoparticles achieved more widespread dispersal in the brains of rats than their cationic counterparts when delivered by CED directly into the corpus callosum or striatum ${ }^{15,16}$ due to their lower affinity for anionic cell surface glycoproteins, and have therefore explored the potential of anionic siRNA nanocomplexes for neurodegenerative diseases. However, with CED, only small volumes $(-5 \mu \mathrm{L} \text { in rat and } \sim 120 \mu \mathrm{L} \text { in pig brains })^{41}$ 


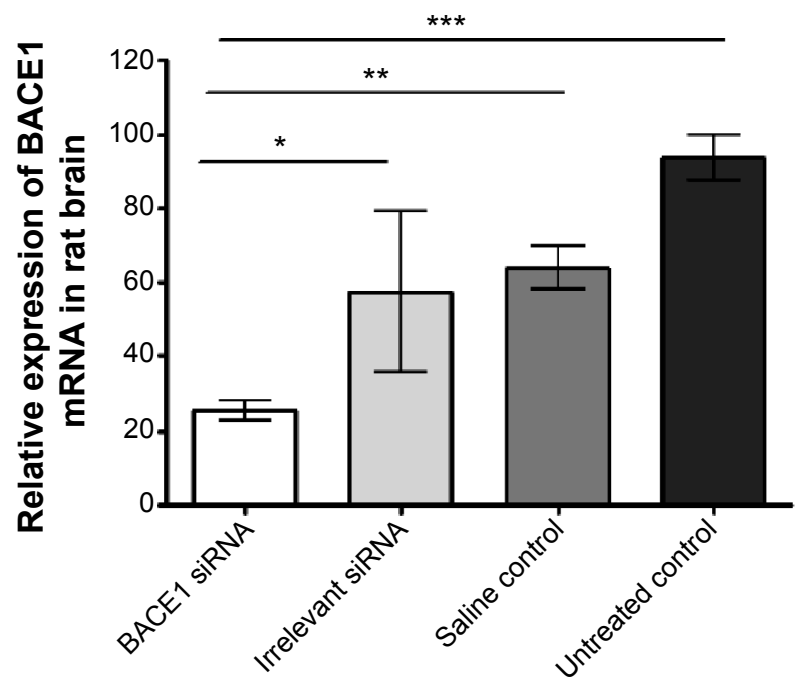

Figure 6 In vivo silencing of BACEI following CED administration of PEGylated concentrated nanoparticles or control saline into rat striatum.

Notes: Concentrated anionic PEGylated PRL nanoparticles containing RVG-9R peptide and BACEI siRNA or irrelevant control siRNA were administered by CED in the striatum of rats, and 48 hours postadministration, tissues were removed for qRT-PCR analysis of siRNA-induced silencing of the BACEI gene. Values are the means of five animals \pm standard deviation ( $n=3$ for the untreated control animals and $n=4$ for the saline control animals) with one-way analysis of variance and Bonferroni's post hoc analysis performed to calculate significant differences $(* P<0.05 ; * * P<0.01 ; * * * P<0.001$ ).

Abbreviations: mRNA, messenger RNA; siRNA, small interfering RNA; CED, convection-enhanced delivery; PEG, polyethylene glycol; PRL, peptide $Y$ or RVG9R, siRNA, liposome LAP2; qRT-PCR, quantitative reverse transcription polymerase chain reaction; $\mathrm{n}$, number.

can be delivered in vivo, therefore concentrating the nucleic acid-containing nanoparticles is necessary in order to deliver higher doses. Further studies using multiple siRNA dosing in order to investigate the duration of the silencing effect may achieve high levels of silencing for a sustained period using systemic or direct administration.

\section{Conclusion}

In this study, we described the development of a method to concentrate cationic and anionic DNA and siRNA nanoparticles to enhance their utility for in vivo applications. Nanoparticles retained their biophysical properties and demonstrated similar silencing efficiency and DNA transfection efficiency in vitro to freshly prepared, nonconcentrated nanoparticles, all without cytotoxicity. Direct delivery into rat brains of $B A C E 1$ siRNA demonstrated significant gene silencing. This method of concentrating nanoparticles for transfection could be used for other formulations, potentially enhancing their utility for in vivo applications.

\section{Acknowledgment}

This work was funded by the Engineering and Physical Sciences Research Council (EPSRC; EP/G061521/1).

\section{Disclosure}

The authors report no conflicts of interest in this work.

\section{References}

1. Couvreur P, Barratt G, Fattal E, Legrand P, Vauthier C. Nanocapsule technology: a review. Crit Rev Ther Drug Carrier Syst. 2002;19(2): 99-134.

2. Glover DJ, Lipps HJ, Jans DA. Towards safe, non-viral therapeutic gene expression in humans. Nat Rev Genet. 2005;6(4):299-310.

3. Love KT, Mahon KP, Levins CG, et al. Lipid-like materials for lowdose, in vivo gene silencing. Proc Natl Acad Sci U S A. 2010;107(5): 1864-1869.

4. Schmidt-Wolf GD, Schmidt-Wolf IG. Non-viral and hybrid vectors in human gene therapy: an update. Trends Mol Med. 2003;9(2):67-72.

5. Semple SC, Akinc A, Chen J, et al. Rational design of cationic lipids for siRNA delivery. Nat Biotechnol. 2010;28(2):172-176.

6. Sheridan C. Gene therapy finds its niche. Nat Biotechnol. 2011;29(2): 121-128.

7. Tagalakis AD, Diakonov IA, Graham IR, et al. Apolipoprotein E delivery by peritoneal implantation of encapsulated recombinant cells improves the hyperlipidaemic profile in apoE-deficient mice. Biochim Biophys Acta. 2005;1686(3):190-199.

8. Yin H, Kanasty RL, Eltoukhy AA, Vegas AJ, Dorkin JR, Anderson DG. Non-viral vectors for gene-based therapy. Nat Rev Genet. 2014;15(8): 541-555.

9. Zhang Y, Satterlee A, Huang L. In vivo gene delivery by nonviral vectors: overcoming hurdles? Mol Ther. 2012;20(7):1298-1304.

10. Anchordoquy TJ, Armstrong TK, Molina MD. Low molecular weight dextrans stabilize nonviral vectors during lyophilization at low osmolalities: concentrating suspensions by rehydration to reduced volumes. J Pharm Sci. 2005;94(6):1226-1236.

11. Grosse SM, Tagalakis AD, Mustapa MF, et al. Tumor-specific gene transfer with receptor-mediated nanocomplexes modified by polyethylene glycol shielding and endosomally cleavable lipid and peptide linkers. FASEB J. 2010;24(7):2301-2313.

12. Manunta MD, McAnulty RJ, Tagalakis AD, et al. Nebulisation of receptor-targeted nanocomplexes for gene delivery to the airway epithelium. PLoS One. 2011;6(10):e26768.

13. Tagalakis AD, Grosse SM, Meng QH, et al. Integrin-targeted nanocomplexes for tumour specific delivery and therapy by systemic administration. Biomaterials. 2011;32(5):1370-1376.

14. Tagalakis AD, McAnulty RJ, Devaney J, et al. A receptor-targeted nanocomplex vector system optimized for respiratory gene transfer. Mol Ther. 2008;16(5):907-915.

15. Kenny GD, Bienemann AS, Tagalakis AD, et al. Multifunctional receptor-targeted nanocomplexes for the delivery of therapeutic nucleic acids to the brain. Biomaterials. 2013;34(36):9190-9200.

16. Tagalakis AD, Kenny GD, Bienemann AS, et al. PEGylation improves the receptor-mediated transfection efficiency of peptide-targeted, self-assembling, anionic nanocomplexes. J Control Release. 2014;174:177-187.

17. Du Z, Munye MM, Tagalakis AD, Manunta MD, Hart SL. The role of the helper lipid on the DNA transfection efficiency of lipopolyplex formulations. Sci Rep. 2014;4:7107.

18. Meng QH, Irvine S, Tagalakis AD, McAnulty RJ, McEwan JR, Hart SL. Inhibition of neointimal hyperplasia in a rabbit vein graft model following non-viral transfection with human iNOS cDNA. Gene Ther. 2013;20(10):979-986.

19. Tagalakis AD, He L, Saraiva L, Gustafsson KT, Hart SL. Receptortargeted liposome-peptide nanocomplexes for siRNA delivery. Biomaterials. 2011;32(26):6302-6315.

20. Tagalakis AD, Saraiva L, McCarthy D, Gustafsson KT, Hart SL. Comparison of nanocomplexes with branched and linear peptides for siRNA delivery. Biomacromolecules. 2013;14(3):761-770.

21. Tagalakis AD, Lee DHD, Bienemann AS, et al. Multifunctional, selfassembling anionic peptide-lipid nanocomplexes for targeted siRNA delivery. Biomaterials. 2014;35(29):8406-8415. 
22. Kenny GD, Villegas-Llerena C, Tagalakis AD, et al. Multifunctional receptor-targeted nanocomplexes for magnetic resonance imaging and transfection of tumours. Biomaterials. 2012;33(29):7241-7250.

23. Couvreur P, Vauthier C. Nanotechnology: intelligent design to treat complex disease. Pharm Res. 2006;23(7):1417-1450.

24. Acebal CC, Simonet BM, Valcárcel M. Nanoparticles and continuousflow systems combine synergistically or preconcentration. TrAC Trends in Analytical Chemistry. 2013;43:109-120.

25. Belliveau NM, Huft J, Lin PJ, et al. Microfluidic synthesis of highly potent limit-size lipid nanoparticles for in vivo delivery of siRNA. Mol Ther Nucleic Acids. 2012;1:e37.

26. Davies LA, Nunez-Alonso GA, Hebel HL, et al. A novel mixing device for the reproducible generation of nonviral gene therapy formulations. Biotechniques. 2010;49(3):666-668.

27. Valencia PM, Farokhzad OC, Karnik R, Langer R. Microfluidic technologies for accelerating the clinical translation of nanoparticles. Nat Nanotechnol. 2012;7(10):623-629.

28. Vauthier C, Cabane B, Labarre D. How to concentrate nanoparticles and avoid aggregation? Eur J Pharm Biopharm. 2008;69(2):466-475.

29. Zelphati O, Nguyen C, Ferrari M, Felgner J, Tsai Y, Felgner PL. Stable and monodisperse lipoplex formulations for gene delivery. Gene Ther. 1998;5(9):1272-1282.

30. Kumar P, Wu H, McBride JL, et al. Transvascular delivery of small interfering RNA to the central nervous system. Nature. 2007;448(7149): 39-43.

31. Cole SL, Vassar R. The role of amyloid precursor protein processing by BACE1, the beta-secretase, in Alzheimer disease pathophysiology. J Biol Chem. 2008;283(44):29621-29625.

32. Golde TE, Dickson D, Hutton M. Filling the gaps in the abeta cascade hypothesis of Alzheimer's disease. Curr Alzheimer Res. 2006; 3(5):421-430.
33. Alvarez-Erviti L, Seow Y, Yin H, Betts C, Lakhal S, Wood MJ. Delivery of siRNA to the mouse brain by systemic injection of targeted exosomes. Nat Biotechnol. 2011;29(4):341-345.

34. Kim SS, Ye C, Kumar P, et al. Targeted delivery of siRNA to macrophages for anti-inflammatory treatment. Mol Ther. 2010;18(5):993-1001.

35. Zadran S, Akopian G, Zadran H, Walsh J, Baudry M. RVG-mediated calpain2 gene silencing in the brain impairs learning and memory. Neuromolecular Med. 2013;15(1):74-81.

36. Gao Y, Wang ZY, Zhang J, et al. RVG-peptide-linked trimethylated chitosan for delivery of siRNA to the brain. Biomacromolecules. 2014;15(3): 1010-1018.

37. Kim JY, Choi WI, Kim YH, Tae G. Brain-targeted delivery of protein using chitosan- and RVG peptide-conjugated, pluronic-based nanocarrier. Biomaterials. 2013;34(4):1170-1178.

38. Bobo RH, Laske DW, Akbasak A, Morrison PF, Dedrick RL, Oldfield EH. Convection-enhanced delivery of macromolecules in the brain. Proc Natl Acad Sci U S A. 1994;91(6):2076-2080.

39. Gill SS, Patel NK, Hotton GR, et al. Direct brain infusion of glial cell line-derived neurotrophic factor in Parkinson disease. Nat Med. 2003;9(5):589-595.

40. Rohn S, Suttkus A, Arendt T, Ueberham U. RVG peptide as transfection reagent for specific cdk4 gene silencing in vitro and in vivo. $J$ Drug Target. 2012;20(4):381-388.

41. White E, Bienemann A, Megraw L, Bunnun C, Gill S. Evaluation and optimization of the administration of a selectively replicating herpes simplex viral vector to the brain by convection-enhanced delivery. Cancer Gene Ther. 2011;18(5):358-369.
International Journal of Nanomedicine

\section{Publish your work in this journal}

The International Journal of Nanomedicine is an international, peerreviewed journal focusing on the application of nanotechnology in diagnostics, therapeutics, and drug delivery systems throughout the biomedical field. This journal is indexed on PubMed Central, MedLine, CAS, SciSearch $®$, Current Contents $\AA /$ Clinical Medicine,

\section{Dovepress}

Journal Citation Reports/Science Edition, EMBase, Scopus and the Elsevier Bibliographic databases. The manuscript management system is completely online and includes a very quick and fair peer-review system, which is all easy to use. Visit http://www.dovepress.com/ testimonials.php to read real quotes from published authors. 